\title{
Children's Imitation is not Always Goal-Directed: Evidence from Goal Clarification Task
}

\author{
Takashi Mizuguchi' ${ }^{1 *}$, Hideaki Shimada ${ }^{1}$, Ryoko Sugimura $^{1}$ and Toshisada Deguchi ${ }^{2}$ \\ ${ }^{1}$ Faculty of Education, University of Shinshu, Nagano, Japan \\ ${ }^{2}$ Faculty of Education, University of Tokyo Gakugei, Tokyo, Japan
}

Received: February 18, 2014; Accepted: March 15, 2014; Published: March 17, 2014

*Corresponding author: Takashi Mizuguchi, Faculty of Education, Shinshu University, 6-ro, Nishinagano, Nagano-380-8544, Japan, E-mail: mizutaka@shinshu-u.ac.jp

\begin{abstract}
The most basic unit of imitation is the ability to translate observed movements into our own movements and reproduce them as our own actions. Goal-directed theories assert that imitation is affected by goals extracted from the observed movements, whereas generalist hypothesis asserts that imitation is affected by the salience of the components rather than the goals. This study developed a new imitation task in order to clarify the role of goals in imitating movement to examine the relative applicability of these two theories to children's imitation. Young children $(\mathrm{N}=55$; mean age $=5: 1$ years, age range $=4$ yr. 1 mo. -6 yr. 0 mo.) were asked to imitate the movements of an experimenter with a toy train. While the children observed the experimenter moving the toy train, they also heard a commentary describing the train's departure and the trip until the train arrives at the station. The commentary emphasized that the train's movements had a start and a goal. We analyzed the children's imitation errors, including whether the color salience of the goal influenced the accuracy of their imitation. The results supported the generalist hypothesis, because the part that was relatively accentuated was more accurately imitated.
\end{abstract}

Keywords: Imitation; Goal-directed theory; Imitation-specific processes of goal selection; Generalist hypothesis; Children

\section{Introduction}

Children may observe movements (usually of people), and in imitation may reproduce these movements in their own actions. One important area of research involves studying the role of the goal in imitation, while another area of research focuses on the salience of the components of the activity that is imitated. People master previous generation's inventions and creations through imitation. Moreover, new practices are introduced into the social group through imitation. Thus, learning through imitation is necessary for adaptation to a specific cultural group [1]. Imitation plays a particularly important role in learning, especially as children begin participating in the cultural group around them [2].

The one of the most important research argument is transformation mechanism from perception to movement in the imitation process (e.g. [3-5]). They can be largely grouped into perspectives that support goal were involved in transformation mechanism and though that do not. The former asserts that imitation is affected by goals extracted from the movements. The latter asserts that imitation is not affected by goals. Imitation is effected by relatively accentuated out of various parts that composed a series of movements. The relatively accentuated parts are not necessary related goals. It is just highly visible parts that composed a series of movements.

The theory of goal-directed imitation (GOADI) posits that imitation is affected by the goal extracted from the movements [6-9]. According to GOADI, observed movements are decomposed into elements of movement patterns, followed by their re composition. GOADI maintains that the decompositionre composition process is strongly affected by goals that are extracted from the movements. For a series of movements composed of several elements, the element that is selected as a goal will attract sufficient attention and be imitated accurately. However, elements that are not selected as a goal may not attract sufficient attention, which may lead to imitation errors. GOADI was initially proposed in research with children, although it was asserted that GOADI is applicable to any age group [9].

A number of research studies have been conducted related to the theory of GOADI. Some studies suggested that there was no fundamental difference in imitation behavior between children and adults $[10,11]$ Prior experience and context influenced children's goal-directed imitation [12-16]. In face-to-face fullbody imitation, children often show mirror responses. When mimicking another person's position, children may imitate as if they are looking in a mirror, or they may use the same hand in the goal-directed imitation [17]; but see [18]. In the first year of life, infants were able to selectively reproduce others' movements toward a goal $[19,20]$. These studies mostly agree in supporting the theory of GOADI.

However, a criticism of GOADI is that the selection of goals is biased, for example when a series of movements was presented; the outcome element (rather than the means by which the elements were performed) was more likely to be selected as a goal [9]. When a target action such as the operation of machinery was presented to children, the action of pressing a switch was 
more likely to be selected as the goal rather than the hand that was used or the trajectory of the movement. This type of goal selection is called imitation-specific processes of goal selection (e.g. [21]). It has been suggested that this is an encoding process for movement imitation that is different from the general encoding process that mediates perception-movement tasks.

The generalist hypothesis posits that imitation is not affected by a goal extracted from the movements, but rather is affected by salience [21,22]. Bird et al. (2007) [21] indicated that when an element was relatively accentuated compared to the other elements that composed a series of movements, then that element was likely to be imitated accurately. Their experiments used a pen-and-cups task, which had been developed as a test of GOADI (e.g. [9]). In Bird et al. adult participants were asked to look at a movement of holding a pen in a hand and moving the pen closer to a cup in front of the person, and then they were asked to imitate the movement. This movement consists of three elements: whether to use the right hand or left (effecter selection), whether to hold the thumb upward or downward while holding the pen (grip selection) and which of the two cups in front to move toward (object selection). If the imitation-specific processes of goal selection are a valid mechanism, then the outcome is more likely to be imitated accurately than the means and thus the number of errors should form a pattern of cup < hand < grip. However, in the results of Bird et al. when an element was relatively accentuated by color, the number of errors for the accentuated element decreased, differing from the error pattern of cup $<$ hand $<$ grip. In other words, imitation-specific processes of goal selection were not supported. The generalist hypothesis was supported because the element was relatively emphasized in the whole, and the movement outcome was not necessarily selected as a goal.

As discussed above, the GOADI and generalist hypothesis do not agree on goal bias. GOADI emphasize the importance of a goal extracted from the movements and imitation-specific processes of goal selection. On the other hand, the generalist hypothesis negates the importance of the goal, and asserts that imitation is mainly affected by elements, which were relatively accentuated in the movements. The reason for this disagreement may involve the characteristics of the pen-and-cups task. In the pen-and-cups task, the participants were presented a series of object manipulations. The three elements that were presented in sequence were clarified visually, however the goal was not clarified because this series of movements was not everyday movements, and thus was relatively incomprehensible. Therefore, the goal was not sufficiently specific, and could not be accurately extracted from the movements.

The present study examines goal bias by using a goal clarification task. The experimenter demonstrates object manipulations to the participants, and at the same time the experimenter provides a commentary about the object manipulations that describes the goal. Specifically in this study with children, the experimenter manipulates a toy train while talking about the train's movements ("Now this train is departing to go to this place" and "the train arrived at this station"). In the commentary, the start, midstream, and goal of the object manipulations are clear and precise. Although the participants in [21] were adults, GOADI was originally proposed in research about children. Therefore the present study used children as participants.

This study analyzes children's imitation responses for elements of movements by using a verbal goal-clarification task. If GOADI is correct, then the goal elements should be imitated more accurately than the other elements. However, if the generalist hypothesis were correct, then the goal elements would not be imitated accurately than the other elements.

\section{Methods}

\section{Participants}

The participants were 55 children, ages 4 yr. 1 mo. to 6 yr. 0 mo. (mean age $=5: 1$ ). Children were randomly assigned into three conditions: control condition $(N=19$, mean age $=5: 2$, age range $=4$ yr. 2 mo. -6 yr. 0 mo.), coloring condition $(N=17$, mean age $=5: 1$, age range $=4$ yr. 1 mo. -6 yr. 0 mo.), and replace condition $(N=19$, mean age $=5: 1$, age range $=4 \mathrm{yr} .1 \mathrm{mo}$. $-5 \mathrm{yr} .11$ mo.). The aims of the study were explained to the parents of the children and the preschoolers, and their consent was obtained.

\section{Materials}

The materials consisted of a green board and a white board, two white plastic containers, and a bilaterally symmetric blue toy train with eight wheels (see Figure 1). The green board was put on top of the white board so that the exposed part of the white board was closest to the experimenter and child, and the green board was further away. The train was initially placed on the white board. The two containers were placed on the green board in the left and right corners furthest from the experimenter and child.

\section{Procedure}

Each child was tested individually in a quiet room. The participant and the experimenter sat on opposite sides of the

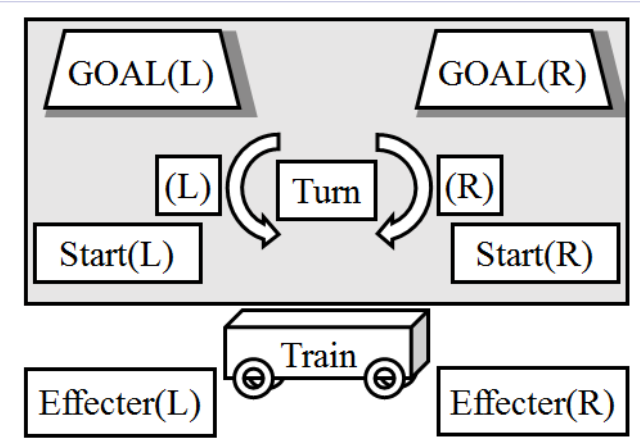

Figure 1: Layout of the materials. The train was a plastic toy train, and the two goals were white plastic containers that were open in the front. Four variable elements were involved in the manipulations of the toy train. The Effecter was the hand (Left or Right) that held and moved the train. The Start was the position (Left or Right) of the train at the start. Turn was whether the train was turned Left or Right 90 degrees. The Goal was the target container for the train. 
table. The experimenter asked the child to play what is called the "mimic game". An action model consisting of simple elements of physically manipulating objects was developed for this study, based on [9]. First, the experimenter picked up the toy train from the white board with either the right or the left hand. Second, the train was placed at the right or left corner on the green board closest to the experimenter and the train was moved to the middle of the green board. Third, the train was turned right or left 90 degrees. Fourth, the train was moved to the destination at the further corner of the green board on the right or left side.

Thus, the action model consisted of four elements: right or left hand (effecter: EF), right or left start point (start: ST), right and left turn (treatment: TR), and right and left destination (goal: GO). In total, 16 variations of the target action were possible $(2 \times 2 \times 2 \times 2=16)$.The sixteen variations were randomized and all variations were demonstrated to each child.

In a practice trial, the experimenter demonstrated some movements to the child and told them "Try to do what I do". Regardless of the child's response, the experimenter said "very good" or "yes".

The experimenter used a playful style of interaction with the child. To begin with, the experimenter pointed at the toy train, and said, "This is a train, do you understand?" Next, pointing at both of the containers, he said, "Both of these are the train stations, do you understand?"Then he said, "Now, this train is departing from this place to go". The experimenter's train was placed on the right or the left side of the green board with his left or right hand, and then moved it to the middle of the green board. The experimenter said, "The train turns around here", and then he turned the train to the right or left 90 degrees with his left or right hand. Then, he asked, "Which station does this train go to? Mmm... the train goes to this station". He then moved the train with his left or right hand into the right or the left station. Next he said, repeating himself, "The train arrived at this station, arrived at this station". The experimenter made verbal comments about the series of train movements to emphasize that the train's movements had a start and a goal. He emphasized that the train arriving at the station was the goal.

The instructions, demonstrations, and commentary were the same in all the conditions. The only thing that was varied was a physical aspect of the goal (see Figure 2). In the coloring condition, a hologram red circle was on one of the white plastic goal containers and a hologram red-x was on the other, which made the goals especially salient. When the experimenter demonstrated the target action, the right position goal had the $\mathrm{x}$-mark and the left position goal had the circle mark. When the child imitated the demonstrated model, the right position goal had the $\mathrm{x}$-mark and the left position goal had the circle mark. In the replace condition, the same marks were used. However, when the experimenter demonstrated the target action, the right position goal had the x-mark and the left position goal had the circle mark. But when the child imitated the demonstrated model, the right position goal had the circle mark and left position goal had the $x$-mark. In other words, in the replace condition, the goals with the red $\mathrm{x}$-mark and circle mark were switched from the left

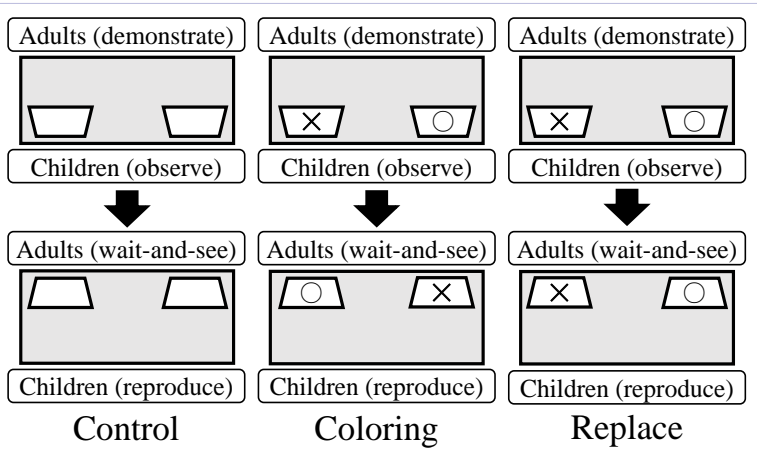

Figure 2: Arrangement of the goal elements in the three conditions. The experimenter and child sat on opposite sides of the table. The top row shows the arrangement for the experimenter's demonstration and the bottom row shows the arrangement for the child's imitation. In the control condition, the containers had no marks. Thus, the demonstration and imitation were not different in the arrangement of the goals. In the coloring condition, the red-x and red circle marked the containers. The demonstration and imitation did not differ in the arrangement of goals. In the replace condition, the goal containers with the red-x and red circle marks were switched in arrangement between the demonstration and imitation. In the demonstration, the experimenter's right side was the $\mathrm{x}$-mark, and the left side was the circle mark. In the imitation, the children's right side was the circle mark, and the left side was the $\mathrm{x}$-mark.

and the right sides from the demonstration to the imitation task. In the control condition, the goals were not visually emphasized with red marks. When the experimenter demonstrated the target action and the child imitated, the right and left position goals were simply the white containers.

\section{Scoring of imitation responses}

Scoring was done using videotapes, which only showed the children's imitative responses. Imitation responses that did not correspond with the protocol for the demonstrated movements were considered error responses. The mean numbers of errors for each condition and action model elements are displayed in Figure 3.

\section{Results}

ANOVA was applied to the number of errors, with condition (Control, Coloring and Replace) as a between subjects factor and the elements of the action model (EF, ST, TR, and GO) as withinsubjects factors. The ANOVA revealed a significant main effect of condition $\left(\mathrm{F}_{2,52}=27.07, p<0.001, \eta^{2}=0.501\right)$, and a main effect of element $\left(\mathrm{F}_{3,156}=22.13, p<0.001, \eta^{2}=0.299\right)$. In addition, there was a significant interaction between condition and element $\left(\mathrm{F}_{6}\right.$, ${ }_{156}=12.33, p<0.001, \eta^{2}=0.314$ ).

A test of simple effects showed that condition was significant for element GO $\left(\mathrm{F}_{2,52}=33.75, p<0.001, \eta^{2}=0.556\right)$. Multiple comparisons by $t$-tests ( $\alpha=0.05$, two-sided test) showed significantly more errors for conditions Control $(p<0.001, d$ $=2.267)$ and Replace $(p<0.001, d=2.425)$ than for Coloring. A test of simple effect showed that element was significant for condition Control $\left(\mathrm{F}_{3,156}=19.04, p<0.001, \eta^{2}=.268\right)$. Multiple comparisons using $t$-tests showed more errors for GO $(p<0.001$, 


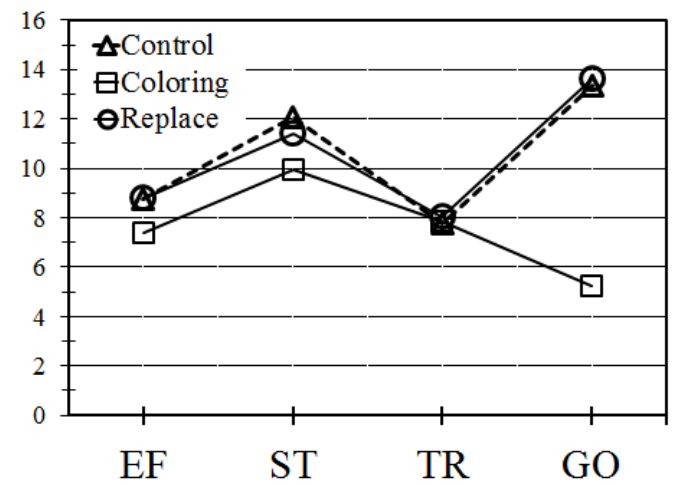

Figure 3: Mean number of errors for each element of the series of movements and for each condition. The elements are EF: Effector; ST: Start; TR: Turn; GO: Goal.

$d=1.094)$ and ST $(p<0.001, d=0.893)$ than for EF, and GO $(p$ $<0.001, d=1.513)$ and ST $(p<0.001, d=1.450)$ than for TR. A test of simple effects showed that element was significant for condition Coloring $\left(\mathrm{F}_{3,156}=10.10, p<0.001, \eta^{2}=0.162\right)$. Multiple comparisons by $t$-tests showed more errors for elements ST than GO ( $p<0.002, d=0.657)$. A test of simple effects showed that element was significant for condition Replace $\left(\mathrm{F}_{3,156}=17.66, p<\right.$ $\left.0.001, \eta^{2}=0.253\right)$. Multiple comparisons by t-tests showed more errors for elements ST ( $p<0.001, d=0.805)$ and GO $(p<0.001, d$ $=1.222)$ than for $\mathrm{EF}$, and more errors for element GO $(p<0.001$, $d=1.586)$ and ST $(p<0.001, d=0.869)$ than for TR.

\section{Discussion}

Numerous research studies have investigated the mechanisms for imitation from perception to movement. Findings from these studies sometimes support and other times do not support the effects of the goal on imitation. GOADI emphasizes goal effects and imitation-specific processes of goal selection (e.g., [9]) When a series of movements was presented, the outcome element was more likely to be selected as a goal, rather than the means by which the elements were performed. In contrast, the generalist hypothesis posits that an element which is relatively accentuated out of various elements that compose a series of movement will be more accurately imitated [21,22].

First, the present study did not support imitation-specific processes of goal selection. The results for the control condition showed that ST and GO had higher error rates than EF and TR. GO emphasized the goal element, which was highlighted in the verbal commentary. Nonetheless, GO was not imitated accurately. In addition, EF had fewer errors of imitation, and was generally imitated accurately. These results do not support the validity of imitation-specific processes of goal selection as proposed by GOADI. Imitation-specific processes of goal selection assert that the outcome element would be imitated accurately, and the means elements would not be imitated as accurately [9]. If imitationspecific processes of goal selection were operational, then outcome elements such as GO would be imitated accurately and means elements such as EF would not be imitated as accurately. However, the control condition yielded results contrary to the view of imitation-specific processes of goal selection. For the reasons given above, goal bias was not supported by our results.

There are other findings to note. The results for the control condition showed more errors of imitation for ST than for TR.EF is obviously applicable means elements, and GO is obviously applicable outcome element. However, ST and TR did not clearly fall into two basic categories. Thus, it is possible that ST has a resemblance to means elements, and TR has a resemblance to outcome elements. If this were the case, then the results for ST and TR would correspond to imitation-specific processes of goal selection, which would predict that means elements would have more errors in imitation than outcome elements. However, the essential research issue involves goal bias. An important concern is validation of whether the encoding process for goals is extracted from movements in imitation. Therefore, the present study analysis of imitation responses uses a goal clarification task. In the results, a large number of errors were found for the clarified goal elements. Furthermore, the GO was the most clear outcome element, and the EF was the most clear means element. If we regarded ST as an exception, the comprehensive results did not support the validity of imitation-specific processes of goal selection.

Second, in the coloring condition, a red circle and red-x marked the containers to visually emphasize the goal elements. Using color to emphasize certain elements that compose a series of movement is the same methodology used in [21,22], which used the pen-and-cups task with adults. [18] used an improved pen-and-cups task with a similar methodology with children. However, these research studies used model movements that were not sufficient to specify the goal. The coloring condition in the present study was implemented to clarify the goal in a concrete way, along with the verbal commentary. The results for the coloring condition showed more errors for ST and TR than for GO, and more errors for ST than EF and TR. The GO element had the lowest rate of errors. In addition to this, the GO element had a higher error rate in the control condition than in the coloring condition.

The reason for the above results is the effect of coloring. That is to say, GO was relatively accentuated out of the various elements that composed the series of movements, so the goal element was accurately imitated. A possible counterargument involves goal bias, that the outcome was more likely to be selected as a goal, and thus the GO element was accurately imitated. However, if this is the case, then the GO element should be imitated accurately not only in the coloring condition but also in the control condition. Thus, these results support the generalist hypothesis [21,22].

Third, we discuss the results for the replace condition. In the replace condition, the goal containers had the red circle and $\mathrm{x}$-mark, which is the same as in the coloring condition. However, the marked positions were not same for demonstration and imitation. In the demonstration, the experimenter's right side had the $\mathrm{x}$-mark and left side had the circle mark containers. However, in the imitation, the children's right side had the circle mark and the left side had the x-mark containers. The children observed the visuospatial movement of the toy train and the two 
goal containers with the marks. Thus, the children selected that which more sufficiently attracted their attention. The replace condition yielded more errors in imitation for ST and GO than for EF and TR. These results show that the GO element had the highest error rate among the four elements. The GO element also had a higher error rate than the control and replace, and the coloring condition.

These results also support the generalist hypothesis. If the goal effect influenced the error rates, then the GO element should not have many errors in the first place. Next, the distinction between the replace condition and the coloring condition involved changing the location of the goal marks between demonstration and imitation. The visuospatial movement of the train was not different in the two conditions. But many more errors were found for the GO element in the replace condition as a result of changing the positions of the marks. Lastly, the reason for the effect of changing the marks was that the marks were relatively accentuated out of the various elements that composed the series of movement. Thus, children gave more sufficient attention to the marks than to the visuospatial movements of the train. This explanation corresponds to the generalist hypothesis.

In closing, this study indicates that when an element is relatively emphasized in the whole, the movement outcome is not necessarily selected as a goal in the children's imitation. Of the two possible theories about imitation mechanisms, one emphasizes a goal effect, while the other does not. However, the previous studies used a series of movement that did not clarify the goal of the movement, such as the pen-and cups task. In the present study, we developed a new imitation task that clarified and concretely represented the goal of the movement by the verbal commentary. That is to say, we examined the goal effect by using a goal clarification task. Thus, we investigated goal effects under conditions that have received little attention in previous studies.

One question worthy of future research is the comparison between children and adults. In this study, we conducted an imitation task with children. The imitation-specific processes of goal selection of GOADI were originally proposed in the context of research with children. Furthermore, a large part of the research studies related to GOADI were conducted with children. However, it is necessary to examine imitation mechanisms in adults as well [21] also reported that error patterns did not differ when a presented target action was converted to movement and when it was explained verbally for adult participants. This indicated that it makes no specific difference whether a target action is converted into movement or is converted into language following visual input. In contrast, [23] used a dual-task method and found that for adults the movement conversion and language conversion involved different mechanisms. Thus, research about imitation by children supports the generalist hypothesis in this study; however imitation by adults did not support all aspects of the generalist hypothesis. Thus, further research with adults is needed using similar methods.

In addition, [22] pointed out that the term "goal" was not clearly defined. They indicated that the term "goal" in GOADI was sometimes extended to cover the notion of physical, endstate goals or mentalistic goals. This matter of the definition of "goal" could complicate finding about the central transformation mechanism from perception to movement in the imitation process. In the present study, the goal elements were physical, end-state goals and mentalistic goals. So the present study did not directly address this matter of the definition of "goal". However, clarifying the definition of "goal" is needed to make an exhaustive model or theory of the imitation mechanism.

\section{Acknowledgement}

This research was supported by a Grant-in-Aid for Japanese Scientific Research (No.21730532) to the first author.

This research presented at the $77^{\text {th }}$ Annual Convention of the Japanese Psychological Association, Sapporo, Japan, in September 2013.

\section{References}

1. Tomasello M (2010) Origins of human communication. MIT Press, Cambridge, Massachusetts, London, England.

2. Tomasello M (1999) The cultural origin of human cognition. The Harvard University Press Cambridge, Massachusetts, USA.

3. Brass M, Heyes C (2005) Imitation: is cognitive neuroscience solving the correspondence problem? Trends Cogn Sci 9(10): 489-495.

4. Heyes C (2009) Evolution development and intentional control of imitation. Philos Trans R SocLond BBiolSci 364(1528): 2293- 2298.

5. Ondobaka S, Bekkering H (2012) Hierarchy of idea-guided action and perception-guided movement. Front Psychol 3: 579.

6. Bekkering H, Wohlschläger A, Gattis M (2000) Imitation of gestures in children is goal-directed. Q J Exp Psychol A 53(1): 153-164.

7. Gattis $M$, Bekkering $H$, Wohlschläger $A$ (2002) The imitative mind: Development evolution and brain bases. Goal-directed imitation. In A N Meltzoff\& W Prinz (Eds) Cambridge University Press, New York, pp. 183-205.

8. Gleissner B, Meltzoff A N, Bekkering H (2000) Children's coding of human action: Cognitive factors influencing imitation in 3-year-olds. Dev Sci 3(4): 405-414.

9. Wohlschläger A, Gattis M, Bekkering H (2003) Action generation and action perception in imitation: An instance of the ideomotor principle. Philos Trans R Soc Lond B Biol Sci 358(1431): 501-515.

10. Mizuguchi T, Suzuki R, Sugimura R, Deguchi T (2010) Imitation of modeled manipulation of objects by children and adults. Percept Mot Skills 110(2): 603-612.

11. Perra O, Gattis M (2008) Reducing the mapping between perception and action facilitates imitation. Bri J Dev Psychol 26(1): 133-144.

12. Diyanni C, Nini D, Rheel W (2011) Looking good versus doing good: which factors take precedence when children learn about new tools? J Exp Child Psychol 110(4): 575-591.

13. Elsner B, Pfeifer C (2012) Movement or goal: goal salience and verbal cues affect preschoolers' imitation of action components. J Exp Child Psychol 112(3): 283-295.

14. Mizuguchi T, Sugimura R, Deguchi T (2009) Children's imitation of movements are goal-directed and context specific. Percept Mot Skills 108(2): 513-523. 
15. Williamson RA, Markman EM (2006) Precision of imitation as a function of preschoolers' understanding of the goal of demonstration. Dev Psychol 42(4): 723-731.

16. Williamson RA, Meltzoff AN, Markman EM (2008) Prior experiences and perceived efficacy influence 3-year-olds' imitation. Dev Psychol 44(1): 275-285.

17. Dunphy Lelii S (2013) Initial investigations into preschoolers' mirrorstyle versus transposed bodily imitation. Can J Exp Psychol 68(1): 3845.

18. Mizuguchi T, Sugimura R, Suzuki R, Deguchi T (2011) Children's imitation is affected by goals but the goals are outstanding action characteristics rather than action outcomes. PSYCH 2: 869-874.

19. Brandone AC, Wellman HM (2009) You can't always get what you want: infants understand failed goal-directed actions. Psychol Sci 20(1): 85-91.

20. Hamlin JK, Hallinan EV, Woodward AL (2008) Do as I do: 7-month-old infants selectively reproduce other's goals. Dev Sci 11(4): 487-494.

21. Bird G, Brindley R, Leighton J, Heyes C (2007) General processes rather than "Goals" explain imitation errors. J Exp Psychol.Hum PerceptPerform 33(5): 1158-1169.

22. Leighton J, Bird G, Heyes C (2010) ‘Goals’ are not an integral component of imitation. Cogn 114(3): 423-435.

23. Mizuguchi T, Sugimura R, Deguchi T (2012) Physical imitation and verbal description of modeled movements engage different encoding processes. Psychol Rep 11(1): 64-74. 